\title{
UNE NOUVELLE ESPÈCE D'ASCAROPHIS (NEMATODA, SPIRURINAE) CHEZ GADUS LUSCUS L. RÉVISION DU GENRE
}

Par Robert Ph. DOLLFUS et Yyonne CAMPANA-ROUGET

Dans un précédent travail, l'un d'entre nous avait discuté la position systématique du genre Ascarophis Van Beneden 1870 et déploré que la structure céphalique n'ait pas été étudiée chez l'espèce-type, A. morrhuæ, mais seulement chez deux espèces assez éloignées, dont les affinités paraissaient douteuses. Depuis lors, nous avons trouvé, dans la collection du Laboratoire d'Helminthologie de l'Ecole Pratique des Hautes-Etudes, une nouvelle espèce d'Ascarophis, parasite de Gadidé ; nous la décrivons plus loin. En outre, nous devons à l'obligeance de Mrs. P. Thomas-Mawson quelques exemplaires non déterminés d'espèces australiennes et antarctiques que nous avons pu rapporter aux espèces suivantes :

A. upeneichtys T. H. Johnston et P. Mawson 1945 chez Upeneichtys porosus (Cul. Val. 1829). St. Vincent Golf, S. Australia.

A. notothenix T. H. Johnston et P. Mawson 1945 chez Notothenia sp. Iles Kerguelen.

A. nototheniæ T. H. Johnston et P. Mawson 1945 chez Trematomus scotti (Boulenger 1907). F 1519 station.

A. nototheniæ T. H. Johnston et P. Mawson 1945 chez Cryodraco antarcticus Dollo 1900 . F 376 station.

A. notothenize T. H. Johnston et P. Mawson 1945 chez Chænodraco wilsoni Tate Regan 1914. F 1543 station.

A. notothenix T. H. Johnston et P. Mawson 1945 chez Trematomus newnesi Boulenger 1902. F 1371 station.

(Les cinq derniers lots ont été recueillis par la B.A.N.Z.A.R. Expedition). Les femelles que nous avons examinées étaient plus grandes que dans la description originale, atteignant 16 à $20 \mathrm{~mm}$. Les œufs étaient pourvus de trois filaments à chaque pôle. Les mâles étaient identiques à la description des spécimens-types.

Anv. de Parasitologie, T. XXXI, N $^{\circ} 4 .-1956$. 
L'étude comparée de ces trois espèces nous a permis de voir que la structure céphalique, en dehors de variations de détail, était suffisamment homogène pour que l'on puisse donner un statut définitif au genre Ascarophis et éliminer les espèces qui ne présentent pas les mêmes caractères. Nous reviendrons là-dessus à propos de la révision du genre.

\section{ASCAROPHIS CRASSICOLLIS nov. sp.}

Matériel : 3 mâles, 6 femelles.

Hôte: Gadus luscus L.

Localisation : estomac.

Localité : La Rochelle (Charente-Maritime).

Date de récolte : 2-4-1941.

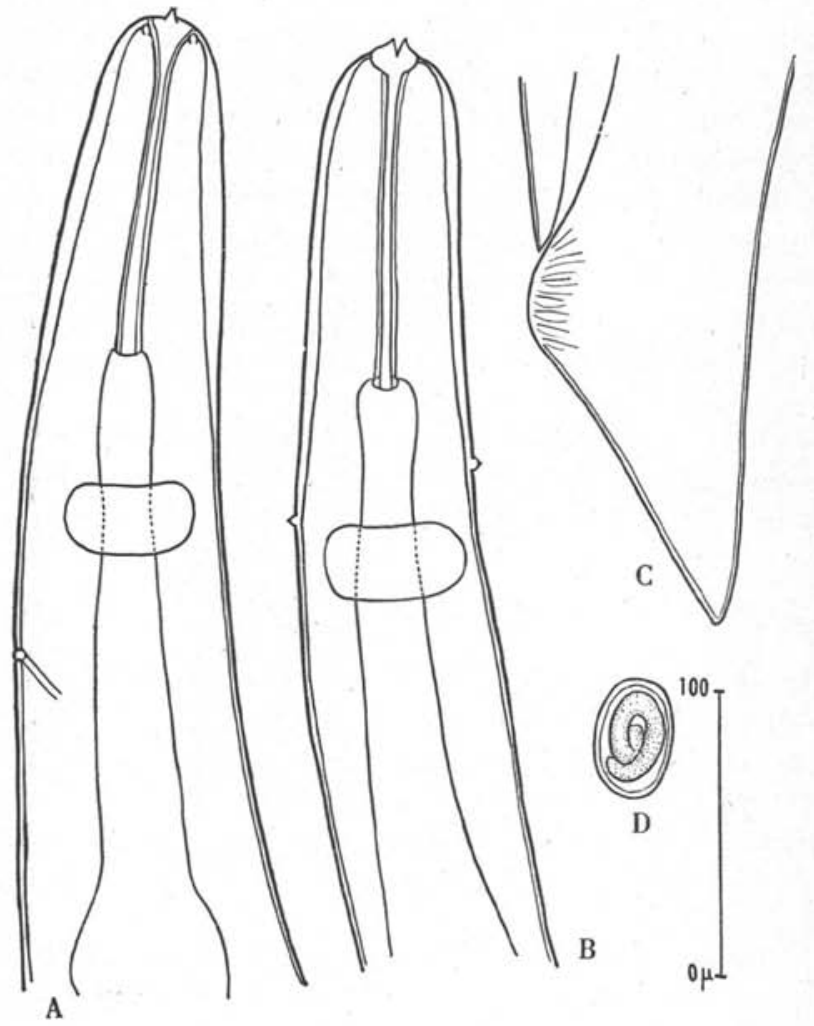

Fig. 1. - Ascarophis crassicollis n. sp.

A, Extrémité antérieure, vue latérale. - B, Extrémité antérieure, vue dorsoventrale. - C, Queue de la femelle. - D, Euf embryonné, in utero. 
Description. - Nématodes filiformes, blanchâtres, de petite taille, s'amincissant progressivement de la moitié du corps à l'extrémité antérieure. Tête arrondie, ornée d'un processus conique médian, bilatéral, en forme de dent. En vue apicale, la bouche, allongée dorso-ventralement, est entourée de deux pseudolèvres en forme de plaquettes, dont la partie interne est élargie et arrondie ; en leur centre se trouve la forte "dent » conique, qui est la seule partie visible en vue longitudinale. Ces lèvres sont en continuité avec la capsule buccale qui est un simple évasement du protorhabdion. Il existe quatre papilles submédianes au cycle externe et deux amphides latérales.

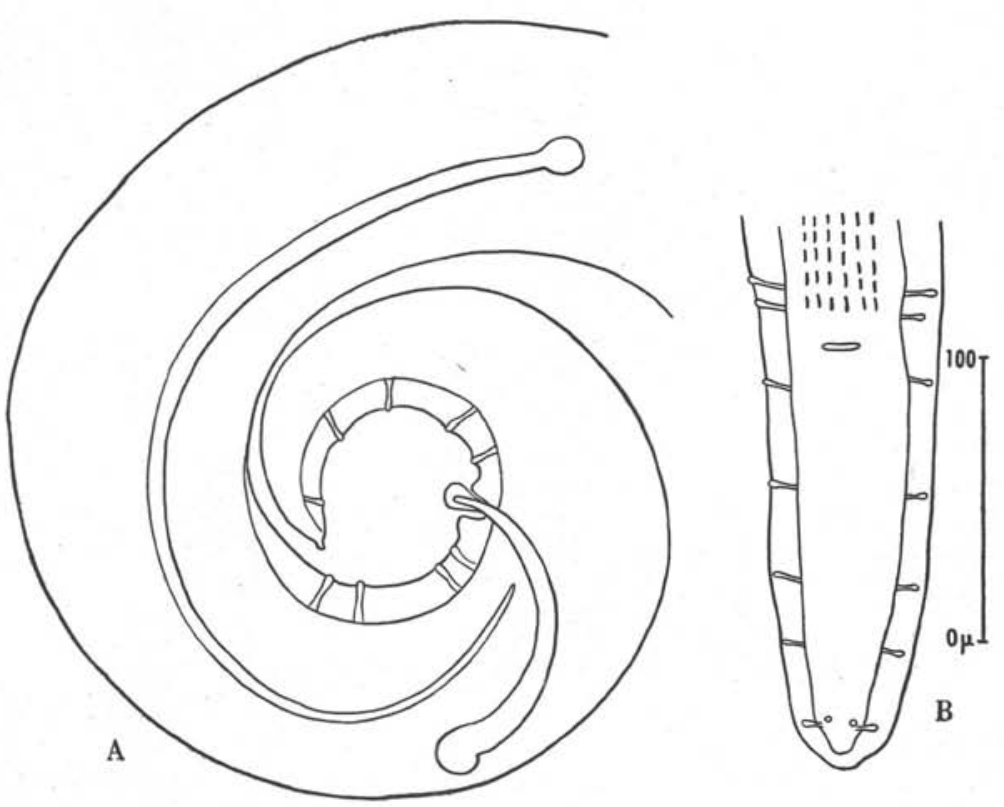

Fig. 2. - Ascarophis crassicollis n. sp.

Extrémité postérieure du mâle : A, profil ; B, face.

Immédiatement en arrière de la tête, la cuticule est épaissie jusqu'aux environs de l'anneau nerveux : il ne s'agit pas ici d'ailes cervicales, mais d'un épaississement régulier tout autour de la région cervicale. Les stries cuticulaires sont visibles, mais peu accentuées.

Mâle : 4,3 à $5,12 \mathrm{~mm}$. de long.

Longueur : $5,12 \mathrm{~mm}$.

Largeur maxima : $0,075 \mathrm{~mm}$. 
Fin de l'œsophage à $1,35 \mathrm{~mm}$. de l'extrémité antérieure.

Protorhabdion et capsule buccale : $110 \mu$.

Esophage antérieur : $190 \mu$.

Esophage postérieur, glandulaire : 1,04 mm.

Anneau nerveux à $172 \mu$ de l'extrémité antérieure.

Diérides à 170 et $150 \mu$ de l'extrémité antérieure.

Pore excréteur à $209 \mu$ de l'extrémité antérieure.

Spicule droit : $128 \mu$.

- gauche : $385 \mu$.

Queue obtuse : $148 \mu$.

Papilles génitales : 4 paires préanales, disposées 2 par 2 ;

5 paires postanales, à peu près équidistantes.

Les phasmides sont au niveau de la $5^{\circ}$ paire.

Les ailes caudales sont moyennement développées et minces.

Sur la face ventrale, en avant du cloaque, la cuticule forme quelques lignes brisées longitudinales, peu marquées.

Femelle : 6,65 à $8 \mathrm{~mm}$.

Longueur : $7,92 \mathrm{~mm}$.

Largeur max. : $150 \mu$.

Fin de l'œesophage à $1,87 \mathrm{~mm}$. de l'apex.

Protorhabdion : $120 \mu$.

(Esophage antérieur : $210 \mu$.

(lisophage glandulaire : $1,51 \mathrm{~mm}$.

Anneau nerveux à $180 \mu$ de l'apex.

Diérides à 145 et $165 \mu$ de l'apex.

Pore excrèteur à $228 \mu$ de l'apex.

Vulve, non proéminente, à $4,49 \mathrm{~mm}$. de l'apex. Vagin dirigé postérieurement.

Queue obtuse : $130 \mu$.

Cúfs embryonnés : $38 \mu \times 30 \mu$ in utero.

Pas de bouchons ni de filaments polaires.

Discussion. - Notre espèce se distingue des autres espèces connues par l'épaississement de sa cuticule cervicale. Elle diffère d'A. morrhux Van Ben. 1870 par la striation beaucoup plus faible de la cuticule, l'absence de filaments polaires sur les œufs, l'inégalité moindre des spicules. Elle diffère d'A. filiformis G. Poljansky 1952, autre espèce parasite de Gadidé, par la taille plus petite, les spicules plus longs et également l'absence de filaments polaires chez l'œuf.

Cette question de présence ou d'absence des bouchons et filaments polaires mérite, à notre avis, d’être précisée : en effet, leur absence, comme beaucoup de caractères négatifs, ne peut signifier de façon absolue qu'il n'y aura pas une apparition plus tardive de ces formations, à la faveur de conditions particulières. Cependant, 
chez tous nos spécimens, les œufs étaient à maturité complète et certains se trouvaient engagés dans l'ovéjecteur, les femelles étaient donc en période de ponte. Ce caractère ne peut en tout cas guère être utilisé dans une clé dichotomique, car, pour plusieurs espèces, la structure de l'œuf n'est pas précisée. Par contre, la présence des filaments est très utile pour la diagnose: s’ils existent, on les retrouve sur tous les œufs utérins embryonnés, tantôt à un seul pôle, tantôt aux deux, en nombre constant pour une espèce donnée.

\section{Révision du genre ASCAROPHIS P. J. Van Beneden 1870}

Nouvelle définition. - Spirurinæ de petite taille, filiformes. Extrémité antérieure amincie, queue obtuse. Bouche allongée dorsoventralement; pseudolèvres moyennement développées, en forme de plaquettes à bord interne élargi et arrondi, portant à la partie médiane un processus conique ou « dent » dirigé vers l'avant. Quatre papilles céphaliques au cycle externe. Mésostome tubulaire et mince, s'évasant antérieurement en un prostome mal défini. CEsophage nettement divisé en deux parties, la première faiblement musculeuse, la deuxième, plus longue, glandulaire. Amphidelphe ; vulve située presque toujours au niveau ou un peu en arrière du milieu du corps. CEufs embryonnés généralement pourvus de filaments à un de leurs pôles ou aux deux. Spicules inégaux; pas de gubernaculum. Quatre paires de papilles préanales, cinq paires postanales. Ailes caudales plus ou moins développées. Face ventrale au-dessus du cloaque ornée de quelques stries longitudinales irrégulières.

Ainsi défini, le genre Ascarophis entre parfaitement dans le cadre de la sous-famille des Spirurinæ, sensu B.G. Chitwood et E. E. Wehr 1934. Le seul amendement à apporter à la définition de la sous-famille concerne le gubernaculum : en effet, Chitwood et Wehr indiquent : « gubernaculum présent ». Or, il n'en existe chez aucun des genres parasites de Poissons et on n'en trouve pas constamment dans les autres genres. Il est done préférable d'écrire : « gubernaculum rarement présent .

Nous allons envisager successivement le statut actuel de l'espècetype, A. morrhuæ, celui des autres espèces toujours connues sous le nom d'Ascarophis, celui des espèces rattachées secondairement à ce genre, et enfin celui des espèces rattachées transitoirement qui doivent être placées ailleurs. 


\section{$1^{\circ}$ Ascarophis morrhuz P. J. Van Beneden 1870}

(p. 56, 92, pl. III, fig. $11 a-11 c$ )

Ce fut longtemps la seule espèce connue, si imparfaitement d'ailleurs qu'il était impossible jusqu’à ces dernières années de la comparer utilement avec les espèces plus récentes; ce n'est, en effet, qu'en 1951, que le mâle est trouvé et, en 1952, qu'une description précise est donnée, toutefois sans vue apicale. Voici un résumé de l'historique qui montre les difficultés rencontrées :

P. J. Van Beneden figure le parasite trouvé chez Gadus morhua L. (Côtes de Belgique) de façon fort imprécise et ne donne qu'un signalement bien vague, sans aucune dimension.

En 1907, W. Nicoll (p. 71, 72, 92-93, 94, pl. IV, fig. 14-16) donne avec un peu plus de détails la description de femelles récoltées chez Melanogrammus xglefinus (L.), qu'il rapporte à A. morrhuæ P.J.V. Ben., ainsi que deux autres exemplaires provenant de Cottus scorpius L. et Hippoglossus hippoglossus (L.), ces trois Poissons venant de St-Andrews (Ecosse). W. Nicoll signale à nouveau, en 1909, A. morrhuæ dans l'estomac de Gadus morhua L., toujours en Ecosse. S'il est probable que les exemplaires trouvés chez des Gadidés soient bien A. morrhuæ P. J. V. Ben., l'identité des espèces trouvées chez des hôtes aussi différents qu'un Chaboisseau ou un Flétan paraît bien douteuse.

En 1933, H. A. Baylis (p. 113-116, fig. 1) décrit de façon beaucoup plus précise deux femelles d'Ascarophis récoltées à Plymouth chez Trigla lineata Gmel. Il n'a que peu d'éléments de comparaison, aussi est-il conduit à penser qu'il n'existe jusque-là qu'une seule espèce, A. morrhuæ P. J. V. Ben., à laquelle il rapporte, non sans quelque hésitation, ses exemplaires. Il laisse également planer quelque doute sur la validité d'une espèce de N. A. Cobb (1928), A. helix, parasite de Dasyatis. Il retrouve la même espèce chez le Grondin en 1933 (Plymouth) et 1939 (South Devon).

H. A. Baylis a le mérite de donner, le premier, une définition du genre, forcément incomplète et basée sur une espèce dont la validité n'est pas certaine. Nous pensons en effet que l'espèce de Baylis n'est pas A. morrhuæ P. J. V. B. : les descriptions récentes d'Ascarophis de Gadidés montrent des différences assez sensibles avec celle de Baylis, surtout en ce qui concerne la taille, alors qu'elles concordent entre elles. Nous donnons ci-dessous les diverses dimensions rapportées.

En 1951, Miss A. R. Gordon (p. 261-262, fig. 1-3) signale enfin la présence d'un mâle et d'une femelle chez Gadus morhua L., en Ecosse, et les décrit assez brièvement. 
Enfin, G. Poljansky, en 1952, donne une description très précise d'A. morrhux P. J. V. Ben. chez Gadus morhua L. et Melanogrammus rglefinus (L.) de la Mer de Barents.

\section{Tableau des dimensions données pour}

A. morrhuæ P.J.V. Ben.

\begin{tabular}{|c|c|c|c|c|}
\hline & Nicol.l 1907 & HAYLIS 1933 & Gordon 1951 & PoLJANSKY \\
\hline $\begin{array}{l}\text { Hòte } \\
\text { Région }\end{array}$ & $\begin{array}{c}\text { Melanogram- } \\
\text { mus aglefinus } \\
\text { (L.) } \\
\text { Ecosse }\end{array}$ & $\begin{array}{c}\text { Trigla lineata } \\
\text { Gmel }\end{array}$ & $\begin{array}{c}\text { Gadus morhua } \\
\text { (L.) } \\
\text { Ecosse }\end{array}$ & $\begin{array}{l}\text { G. morhua (L.) } \\
\text { M. aglef. (L.) } \\
\text { Mer de Burents }\end{array}$ \\
\hline 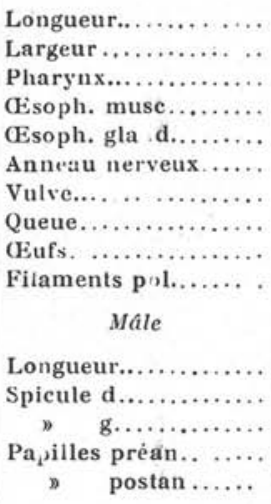 & $\begin{array}{l}6-8 \mathrm{~mm} \\
0,08-0,09\end{array}$ & $\begin{array}{c}\mathrm{t1}, 2-13,2 \mathrm{~mm} . \\
0,085 \cdot 0,11 \\
0,150 \\
0,47 \\
2,38 \\
0,21 \\
7,28, \AA \text { de } 1 \cdot \mathrm{E} . \mathrm{a} . \\
0,065-0,07 \\
3740 \times 2225 \mu \\
0\end{array}$ & $\begin{array}{c}5,5 \\
8,10\left(\sigma^{7}\right) \\
0,27 \quad b \\
1,10 \quad b \\
0,136 \\
44-47 \times 2225 \mu \\
2 \text { à } 1 \text { póle }\end{array}$ & $\begin{array}{c}5,35-7,24 \\
0,072-0,092 \\
0,25 \\
0,908-1,378 \\
0,135 \\
2,8-4,24 \\
\\
41-45 \times 22-27 \mu \\
2 \text { à } 1 \text { pôle } \\
\\
3,68-4,29 \\
0,08-0,083 \\
0,50-0,59 \\
4 \\
5\end{array}$ \\
\hline
\end{tabular}

En résumé, l'espèce-type, A. morrhuæ P. J. Van Ben. 1870, est actuellement bien définie. Nous croyons qu'il faut en exclure les spécimens de W. Nicoll provenant de Cottus scorpius L. et d'Hippoglossus hippoglossus (L.), qui, n'étant ni décrits, ni figurés, ne peuvent être que signalés comme Ascarophis sp. Nous croyons également que l'espèce de $\mathrm{H}$. A. Baylis doit être mise à part; le mâle n'est pas connu, mais la description de la femelle est suffisante, et nous proposons le nom d'A. Baylisi nom. nov. (= A. morrhuæ H. A. Baylis 1933).

\section{$2^{\circ}$ Autres espèces (groupées selon leur répartition géographique)}

A. helix N. A. Cobb 1928. Chez Dasyatis centroura (Mitchill 1815). Amérique du Nord (Massach.). Mâle inconnu. Une seule femelle. $S p$. inquirenda. 
A. cestus B. G. Chitwood 1934. Chez Colorhynchus sp., Porto-Rico, Am. centrale. Mâle inconnu.

A. sebastodis L. S. Olsen 1952. Chez Sebastodes caurinus (J. Richardson 1845). Ile San Juan, Washington. Mâle inconnu.

A. arctica G. Poljansky 1952. Chez Zoarces viviparus (L. 1758). Mer de Barents.

A. filiformis G. Poljansky 1952. Chez Gadus morhua L. 1758 et Melanogrammus aglefinus (L.). Mer de Barents.

A. crassicollis n. sp. Chez Gadus luscus L. 1758. France (Charente-Maritime).

A. baylisi nom. nov. pour A. morhua H. A. Baylis 1933. Chez Trigla lineata Gmel. 1789. Sud de l'Angleterre. Mâle inconnu.

A. australis T. H. Johnston et P. Mawson 1944. Chez Threpterius maculosus J. Richardson 1850. Australie du Sud, Cape Borda.

A. cooperi J. et M. 1945. Chez Platycephalus bassensis Cul. Val. 1829. Australie du Sud, Rapid Bay.

A. upeneichtys J. et M. 1945. Chez Upeneichtys porosus (Cuv. Val. 1829). Austr. du Sud, Albany, St. Vincent Golf.

A. notothenix J. et M. 1945. Chez de nombreux Nototheniidx. Antarctique et subantarctique.

A. chalinurze J. et M. 1945. Chez Chalinura ferrieri T. Regan 1913. Antarctique.

A. licodichtys J. et M. 1945. Chez Licodichtys antarcticus Pappenheim 1911. Antarctique. Mâle inconnu. Une femelle détériorée. Sp. inquir.

A. $s p$. R.-Ph. Dollfus et Y. Campana-Rouget 1956. Chez Latimeria chalumnæ J. L. B. Smith 1939 comorensis, Comores. Mâle inconnu.

\section{$3^{\circ}$ Espèces rattachées secondairement à Ascarophis}

Cyclozone acipenserina V. Dogiel 1932. Chez Acipenser sturio L. 1758, A. stellatus Pallas 1771, d'après B. G. Chitwood 1934.

Capillospirura ovotrichuria K. Skrjabin 1924. Chez Acipenser güldenstädti Brandt 1833, A. ruthenus L. 1758, A. stellatus Pallas 1771, Huso huso (L. 1758). Bassin de la Volga. D’après G. Poljansky 1952.

Pseudocystidicola skrjabini E. M. Layman 1933. Chez Acipenser baeri Brandt 1869, Cottus kneri Dybowski 1874. D'après G. Poljansky 1952.

Cystidicola skrjabini St. Markowski 1938. Chez Zoarces viviparus (L. 1758). Baltique. L'auteur identifie son espèce à celle de Layman. Georges Poljansky pense que c'est inexact; il en fait un Ascarophis, sans toutefois l'assimiler à une espèce donnée. Or, il semble bien, malgré l'absence de mâle, qu'elle soit identique à $A$. arctica décrite par Poljansky 1952 chez le même hôte. 
Rhabdochona girellæ S. Yamaguti 1935. Chez Girella punctata Gray 183035. Japon. D'après Y. Campana-Rouget 1955.

Rhabdochona gymnocranii S. Yam. 1935. Chez Gymnocranius griseus (Temm. et Schlegel 1843). Japon. Mâle inconnu. D’après Y. Campana-Rouget 1955 .

\section{Glé de détermination des espèces d'Ascarophis dont le mâle est connu}

1 (26) Pas de dilatation cuticulaire cervicale.

2 (11) Cuticule marquée par des stries profondes.

3 (8) Stries profondes seulement dans la région céphalique.

4 (5) Esophage court $(<1 \mathrm{~mm}.) \ldots \ldots \ldots \ldots \ldots \ldots \ldots \ldots$. cestus.

5 (4) (Esophage long ( $>1,5 \mathrm{~mm}$.).

6 (5) Vulve post-équatoriale.................. acipenserina.

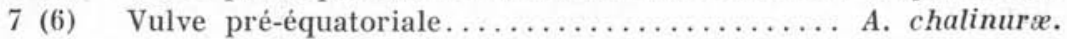

8 (3) Stries profondes sur tout le corps, sauf la région céphalique.

9 (10) Ailes caudales ornées de petits tubercules.

(Eufs pourvus de 3 filaments à chaque pôle..... A. nototheniæ.

10 (9) Ailes caudales lisses. CEufs pourvus de 2 filaments à un

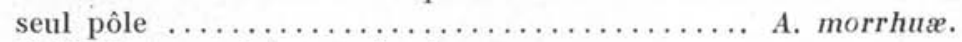

11 (2) Cuticule sans stries profondes.

12 (25) Protorhabdion long $(>100 \mu)$.

13 (14) Spicule droit $=1 / 8^{\circ}$ du spicule gauche. Cufs pourvus de 5 filaments ou plus à chaque pôle............. arctica.

14 (13) Spicule droit $=1 / 3$ ou $1 / 4$ du spicule gauche.

15 (16) Vulve équatoriale............................

16 (15) Vulve post-équatoriale.

17 (18) Vulve très postérieure ................... australis.

18 (17) Vulve au début du $1 / 3$ postérieur.

19 (22) Apex du spicule gauche de structure simple.

20 (21) Gufs pourvus de 2 filaments à chaque pôle....... A. skrjabini.

21 (20) Eufs pourvus de 2 filaments à un seul pôle....... A. filiformis.

22 (19) Apex du spicule gauche de structure complexe.

23 (24) Apex pointu, armé d'une épine............... cooperi.

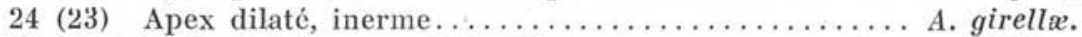

25 (2) Protorhabdion court $(<50 \mu) \ldots \ldots \ldots \ldots \ldots \ldots$ A. upeneichtys.

26 (1) Dilatation cuticulaire cervicale............. crassicollis.

\section{$4^{\circ}$ Espèces exclues du genre Ascarophis}

Ce sont les espèces du genre Metabronema W. Yorke et P. Maplestone 1926. B. G. Chitwood n'ayant pas reconnu la validité de ce genre, les avait reportées dans Ascarophis. Le genre Metabronema 
a été rétabli par Choquette (1948), puis par l'un de nous (Y. Campana-Rouget, 1955) avec un sens restrictif : il ne comprend plus que des espèces parasites de Poissons de mer ou de migrateurs ; il est caractérisé par la présence de pseudolèvres très développées et de revêtements chitinoïdes épais intéressant le protorhabdion, la capsule buccale et le pourtour oral. Il est représenté par :

M. magna (E. L. Taylor 1925). Espèce-type. Vessie natatoire de Trachurus declivis (Jenyns 1842), cavité générale de Sparus sp., Australie.

M. uvardlei (E. M. Smedley 1934). Intestin de-Scorpænichtys marmoratus (Ayres 1854). Côte Pacifique du Canada.

M. caranxi M. Tubangui et V. Masilungan 1937. Cav. gén. de Caranx speciosus (Forskâl 1775). Philippines.

Il convient d'y ajouter certaines des espèces de Ts. Fujita :

M. kosugii Ts. Fujita 1939. Cav. gén. de Salvelinus kundscha (Pallas 1811). Japon.

M. ishii Ts. Fujita 1939 (= M. salvelini F. 1939). Intestin de Salvelinus kundscha (Pallas 1811).

(Ces deux espèces sont très proches l'une de l'autre et peut-être synonymes; elles se distinguent des autres par la présence de 8 à 10 paires de papilles préanales au lieu de 4).

M. amemasu Ts. Fujita 1939. Intestin de Salvelinus kundscha (Pallas 1811). Mâle inconnu.

Les autres espèces décrites par Fujita sous le nom de Cystidicola ou Metabronema sont à peu près impossibles à classer sans revoir les types, car il y a très souvent discordance entre le texte et les figures : pour Cystidicola chitosensis Ts. Fujita 1940, par exemple, on lit que le pharynx mesure $0,10 \mathrm{~mm}$. et que l'anneau nerveux est à $1,4 \mathrm{~mm}$. de l'extrémité antérieure ; or, d'après la figure, ces dimensions sont respectivement de $0,05 \mathrm{~mm}$. et $0,15 \mathrm{~mm}$., soit 2 fois et 10 fois plus petites. Pour $M$. amemasu Ts. Fujita 1939, on lit : «pharynx remarquablement court et étroit »; or, il est un peu plus long et nettement plus large que chez $M$. kosugii et $M$. ishii décrits en même temps.

Nous croyons, mais sans pouvoir l'affirmer, que Cystidicola iwana Ts. Fujita 1928 est bien un Cystidicola, car il y a 20 paires de papilles préanales couplées en partie (Laurent Choquette, 1948, en fait un Metabronema).

Nous pensons que $\boldsymbol{M}$. oncorhynchi Ts. Fujita 1939 est également un Cystidicola pour les mêmes raisons. Inversement, $C$. chitosensis 
Ts. Fujita 1940 est probablement un Metabronema (forte chitinisation buccale et papilles non couplées). M. laticauda et $C$. brevicauda Ts. Fujita 1939 pourraient bien être des Ascarophis, si l'on en juge par la longueur et l'étroitesse du pharynx.

Il reste encore à classer un petit nombre d'espèces, placées antérieurement dans les Metabronema, mais qui s'en éloignent par un certain nombre de caractères : pseudolèvres peu développées, pharynx étroit à parois minces, capsule buccale mal définie, ouverture buccale non renforcée. L'espèce-type de ce groupe, décrite en 1931 par Chandler sous le nom de Cystidicola harusoodi, après avoir passé sucessivement dans plusieurs genres, avait été étudiée par B. G. Chitwood sous le nom d'Ascarophis en 1950 ; la vue apicale qu'il en donnait paraissait assez différente de celle qu'il représentait en 1934 pour Ascarophis cestus B. G. Chitwood. Il s'agissait de savoir laquelle de ces deux figures correspondait réellement au genre Ascarophis. Or, nous avons étudié les structures céphaliques d'A. crcssicollis, A. notothenix et A. upeneichtys : toutes trois sont identiques et tout à fait comparables à celle d'A. cestus. Celle d'A. harwoodi semblait au contraire se rapprocher du Spinitectus représenté par Chitwood. (Nous verrons plus loin ce qu'il faut penser de ces structures céphaliques). Les Spinitectus étant ornés de rangées d'épines, il était difficile de placer parmi eux des espèces dépourvues de ces ornements. Nous préférons donc reprendre l'ancien genre créé par M. S. Skinker en 1931, précisément pour Cystidicola harwoodi A. C. Chandler 1931 et Cystidicola fischeri L. Travassos, P. de T. Artigas et Cl. Pereira 1928; il avait été mis en synonymie avec Metabronema par H. A. Baylis en 1934, car les caractères donnés paraissaient insuffisants. La définition de M. S. Skinker était la suivante :

«Thelaziidæx: Bouche avec des lèvres latérales et une paire de structures en forme de dent ou de papille; vestibule à paroi chitineuse; œsophage long. Mâle : extrémité postérieure enroulée en spirale, queue arrondie au bout, ailes caudales étroites et supportées par 4 paires de papilles préanales simples et 4 ou 5 paires de papilles postanales; spicules très inégaux. - Femelle : queue courte et obtuse ; vulve approximativement équatoriale ou postérieure au milieu du corps. Ovipare.

Espèce-type : C. fischeri (Trav., Art. et Per. 1928). ».

$\mathrm{Si}$ cette définition est exacte, sauf en ce qui concerne la classification, elle ne précise pas les points importants. Il faut y ajouter les caractères que nous avons donnés ci-dessus, soit: vestibule étroit, à parois minces, ouverture et capsule buccales sans renfor- 
cements chitinoïdes importants, pseudolèvres peu développées. Parasites de l'estomac ou de l'intestin de Poissons d'eau douce.

Ce genre est en réalité beaucoup plus proche de Spinitectus que de Metabronema.

Espèces entrant dans le genre Cystidicoloides Skinker 1931:

C. fischeri (Travassos, Artigas et Pereira 1928). Skinker 1931. Espècetype.

(= Cystidicola fischeri Trav., A. et P. 1928).

(= Metabronema fischeri Baylis 1934).

Estomac de Salminus maxillosus (Cuv. et Val.). Brésil.

C. harwoodi (A. C. Chandler 1931) Skinker 1931.

(= Cystidicola harwoodi Chandler 1931).

(= Metabronema harwoodi Baylis 1934).

(= Ascarophis harwoodi Chitwood 1950).

(= Metabronema canadense Skinker 1931).

Estomac de Salvelinus fontinalis (Mitchill 1815). Etat de New-York, Canada.

C. salvelini (Fujita 1920).

(= Spiroptera salvelini Fujita 1920).

(= Cystidicola salvelini Fujita 1928).

(= Metabronema salvelini Baylis 1935).

Intestin de Salvelinus malma, Salvelinus kundscha. Japon.

C. truttz (Baylis 1935).

(= Metabronema truttre Baylis 1935).

Intestin de Salmo trutta L. Angleterre.

C. prevosti (Choquette 1951).

(= Metabronema prevosti Choquette 1951).

Intestin de Ameiurus nebulosus (Le Sueur). Canada.

Choquette, en 1948, dans une révision du genre Metabronema, avait mis en synonymie les trois avant-dernières espèces citées ci-dessus. Il est certain qu'elles présentent des affinités étroites, mais il serait surprenant qu'une seule espèce ait une répartition géographique aussi dispersée; aussi est-il préférable de conserver ces trois espèces, en attendant une nouvelle étude des spécimenstypes.

Nous pensons pouvoir ajouter à cette liste l'Ascarophis murrayensis de T. H. Johnston et P. Mawson 1947, parasite de Plectroplites ambiguus (J. Richardson 1844), Australie du Sud. Nous y . mettons cependant quelques réserves, car ce Nématode a été décrit brièvement et, fort malencontreusement, le dessin représentant la partie antérieure a été omis. 


\section{Conclusions}

L'étude des Spiruridés parasites de Poissons est extrêmement difficile, non en ce qui concerne la description de telle ou telle espèce, mais bien son attribution à un genre donné : on ne peut s'occuper d'une espèce connue depuis plus de 20 ans sans découvrir qu'elle a été classée successivement dans trois genres différents, au moins ; quant aux genres, ils ont été à plusieurs reprises mis en synonymie ou rétablis, sauf Cystidicola Fischer 1798 et Spinitectus Fourment 1883 ; encore, pour ce dernier, Chitwood et Wehr (1934) estiment que, sauf en ce qui concerne les épines, ce genre peut être considéré comme synonyme d'Ascarophis Van Ben. 1870. Cependant, les espèces de Spinitectus n'ont pas subi de changements, à cause de ce caractère évident qu'est la présence de rangées d'épines ; mais, si l'on avait utilisé les mêmes critères que pour les autres genres, les mêmes indécisions se seraient inévitablement reproduites.

Cet état de choses ne provient que pour une faible partie de l'imprécision des descriptions anciennes (le même problème se retrouve chaque fois que l'on étudie un Helminthe et ne constitue pas ici un cas particulier). Il est surtout lié au fait que, sauf pour les caractères communs à tout le groupe, aucun des caractères généralement utilisés dans une diagnose générique n'est ici absolument constant. En voici quelques exemples :

Le nombre de papilles préanales est très souvent de quatre paires, comme chez beaucoup d'autres Spirurides. Chez Cystidicola, il est de 8 à 20 paires, dont certaines sont groupées deux par deux. Ce caractère devrait être excellent, mais, chez Metabronema, deux espèces, $M$. kosugii T. Fujita 1939 et $M$. ishii T. Fujita 1939, ont 8 et 10 paires de papilles; chez Spinitectus, une seule espèce, S. yorkei Travassos, Artigas et Pereira 1928, en possède 15 paires ; la seule différence avec Cystidicola est que ces papilles ne sont jamais couplées.

La position de la vulve a pu être utilisée dans une certaine mesure, mais, si elle est généralement prééquatoriale chez Metabronema, équatoriale chez Cystidicola, postéquatoriale chez Spinitectus, Ascarophis et Cystidicoloides, il y a un certain nombre d'exceptions.

L'étude de la structure céphalique en vue apicale apporte des renseignements intéressants, mais peut-être difficiles à interpréter si l'on n'a pas d'éléments de comparaison: dans tout le groupe, 
les pseudolèvres sont réduites à un seul lobe médian, très développé chez Metabronema, rudimentaire chez Cystidicola, moyen chez les trois autres. Ce lobe porte une dent dirigée vers l'avant, bien développée chez Ascarophis, peu visible chez les autres en général. Nous n'avons pas encore pu étudier de Metabronema, mais nous avons examiné trois espèces d'Ascarophis, un Cystidicola, un

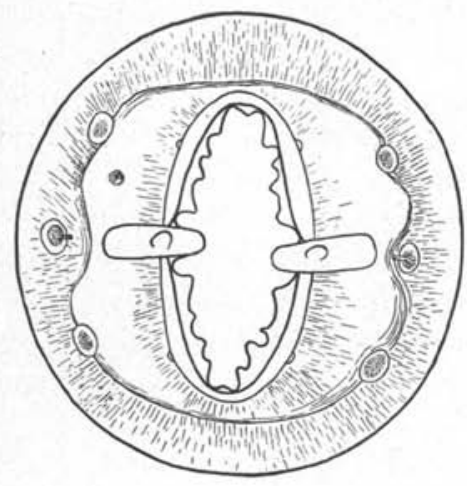

A

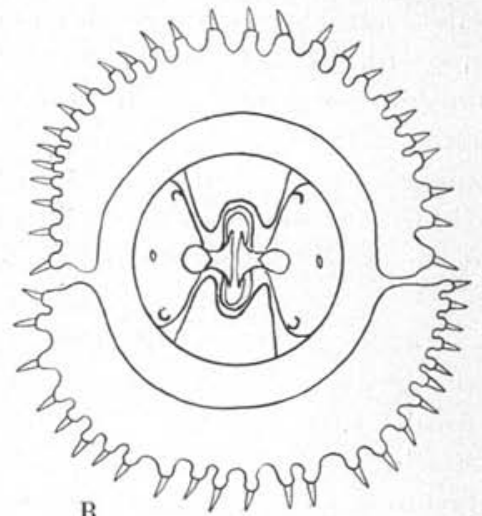

B
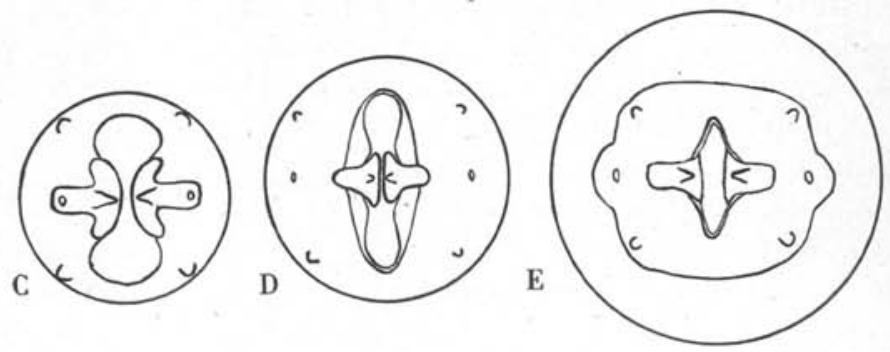

FIG. 3. - Extrémités céphaliques, vue apicale.

A, Cystidicola stigmatura (Jos. Leidy 1886) (dessin inédit de Camille Desportes). - B, Spinitectus sp. - C, Ascarophis crassicollis n. sp. - D, Ascarophis nototheniæe T. H. Johnston et Patricia Mawson 1945. - E, Ascarophis upeneichtys T. H. Johnston et Patricia Mawson 1945.

Cystidicoloides, et plusieurs Spinitectus: Cystidicola stigmatura présente un lobe médian distinct, mais peu développé, à bord interne non élargi, et des dents, ou plutôt des lames disposées tout autour de la bouche ; ce dernier caractère n'étant pas signalé chez les autres espèces, on ignore à l'heure actuelle s'il est générique ou seulement spécifique.

Cystidicoloides et Spinitectus ont exactement la même structure céphalique, se distinguant de celle d'Ascarophis par la présence 
de lèvres chitinoïdes dorsale et ventrale et la réduction des dents latérales à peine visibles ; l'existence de lèvres dorso-ventrales (ou interlabia pour certains auteurs) les rapproche des Habronematinae, qui possèdent de telles formations. Choquette (1951) ne les a pas vues chez $C$. prevosti, mais son matériel était en mauvais état.

Un bon caractère différentiel nous paraît être la structure du pharynx et de la capsule buccale, bien que, là encore, l'interprétation personnelle puisse jouer un certain rôle et que l'on soit forcé d'être un peu trop schématique : le maximum de chitinisation se rencontre chez Metabronema où le pharynx, relativement court, de diamètre assez grand, est renforcé par des parois épaisses qui se prolongent le long de la capsule buccale bien délimitée ; le pourtour buccal et les pseudolèvres sont également renforcées par des épaississements chitinoïdes. Chez Cystidicola, le pharynx est du même type, la capsule buccale est encore bien limitée, mais les renforcements buccaux ont disparu. Chez Spinitectus, le pharynx, assez court, est étroit et entouré d'une paroi mince, la capsule buccale n'est plus marquée que par un évasement du protorhabdion et se trouve ainsi mal limitée. Chez Ascarophis et Cystidicoloides, le pharynx est long, les autres caractères sont les mêmes que pour Spinitectus.

Il semblerait qu'en groupant les caractères énumérés ci-dessus, on puisse arriver à une détermination. Mais, ce qui complique encore les choses, c'est que ces caractères ne vont pas forcément de pair. On a l'impression que l'évolution de ce groupe se fait de façon absolument anarchique, sans que l'on puisse savoir quel est le type primitif ou évolué, ni comparer avec ce que l'on voit chez les Spirurides des autres Vertébrés.

Nous pouvons cependant distinguer deux groupes dont les chefs de file sont Cystidicola d'une part, Ascarophis de l'autre. C'est entre ces deux genres que les différences sont les plus marquées, tant du point de vue morphologique que du point de vue biologique : le premier est un parasite tissulaire à peu près strict (on le rencontre parfois dans l'œsophage, mais généralement en même temps dans la vessie natatoire) ; on le trouve chez des Poissons d'eau douce ou des migrateurs récoltés en rivière; le nombre de papilles préanales est toujours élevé et comporte plusieurs paires couplées, le pharynx est relativement court, fortement chitinisé, la capsule buccale est marquée. Ascarophis est strictement un parasite intestinal, presque toujours de Poissons marins, sauf un petit groupe parasite d'Esturgeons ; il y a toujours quatre paires de papilles préanales, le pharynx est long et étroit, à parois minces, la capsule buccale est mal limitée. 


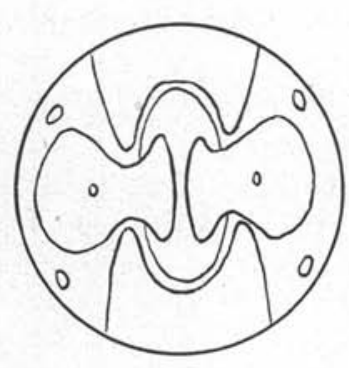

FIG. 4. - Cystidicoloides truttex (Baylis 1935). Extrémité céphalique, vue apicale.

Dans le groupe Cystidicola, entre le genre Metabronema, qui se distingue de Cystidicola par le développement de ses pseudolèvres et sa chitinisation buccale plus accentuée, mais en est proche par son affinité tissulaire, moins stricte toutefois, puisque certaines espèces sont rencontrées dans l'intestin, et la possibilité d'avoir un nombre de papilles supérieur à quatre.

Dans le groupe Ascarophis se rangent tous les autres genres: Spinitectus, parasite de Poissons d'eau douce ou d'Anguilles (un seul, S. oviflagellis Fourment 1883, a été trouvé chez Merlangus merlangus (L.), et on peut se demander s'il n'y a pas eu une erreur d'étiquette). Il est très proche d'Ascarophis, mais est couvert de rangées d'épines, ce qui est un bon caractère générique.

Cystidicoloides, parasite intestinal de Salmonidés d'eau douce ou migrateurs, très proche de Spinitectus, mais sans épines.

Parascarophis Y. Campana-Rouget 1955, parasite intestinal de Sélacien, qui possède deux petites dents sur chaque pseudolèvre et une cuticule céphalique renflée en forme de capuchon. La vulve est nettement prééquatoriale chez l'espèce-type, $P$. sphyrnæ CampanaRouget 1955 .

Ce deuxième groupe se rapprocherait un peu des Acuariidx par certains traits : pharynx mince et long, présence fréquente d'ornements cuticulaires tels qu'épines ou collerettes ; mais il s'en éloigne par son peu d'affinité pour les tissus; au contraire, le groupe Cystidicola, qui présente cette affinité, est plus proche morphologiquement du genre Spirura.

Nous donnons ci-dessous un schéma résumant les principales particularités des différents genres :

\begin{tabular}{|c|c|c|c|c|c|}
\hline \multicolumn{2}{|c|}{$\begin{array}{l}\text { Parasites tissulairiss } \\
\text { Pharynx large }\end{array}$} & \multicolumn{4}{|c|}{$\begin{array}{l}\text { PARASITES DU TUBE DIGESTIF } \\
\text { PแARYNX Éา RคIT }\end{array}$} \\
\hline $\begin{array}{c}\text { Poissons } \\
\text { de rivière } \\
\text { Salmonidés }\end{array}$ & $\begin{array}{c}\text { Poissons } \\
\text { de mer } \\
\text { Salmonidés }\end{array}$ & $\begin{array}{l}\text { Rivière } \\
\text { Anguillidæe }\end{array}$ & $\begin{array}{l}\text { Rivière } \\
\text { Salmonidés }\end{array}$ & $\begin{array}{c}\text { Mer } \\
\text { Acipenseridès }\end{array}$ & $\begin{array}{c}\text { Mer } \\
\text { Sélaciens }\end{array}$ \\
\hline $\begin{array}{l}\text { Bouche parfois } \\
\text { armée de dents. } \\
\text { Lèvres rudimen- } \\
\text { taires. Plus de } 4 \\
\text { pairesde papilles } \\
\text { préanales. }\end{array}$ & $\begin{array}{l}\text { Bouche inerme } \\
\text { Lèvres déve- } \\
\text { loppées. Papil- } \\
\text { les variables. }\end{array}$ & $\begin{array}{c}\text { Rangées } \\
\text { d'épines sur } \\
\text { tout le corps. }\end{array}$ & $\begin{array}{l}\text { Pas d'èpines. } \\
\text { Pas de dent } \\
\text { développéesur } \\
\text { les lèvres. }\end{array}$ & $\begin{array}{l}\text { Pas d'épines. } \\
\text { Une dent } \\
\text { développéesur } \\
\text { chaque lèvre. }\end{array}$ & $\begin{array}{l}\text { Pas d'epines. } \\
2 \text { dents sur } \\
\text { chaque lèvre. } \\
\text { Capuchon } \\
\text { céphalique }\end{array}$ \\
\hline Cystidicola & Metabronema & Spinitectus & Cystidicoloides & Ascarophis & Parascarophis \\
\hline
\end{tabular}


Un fait singulier à constater est le nombre impressionnant de Poissons migrateurs que l'on trouve parmi les hôtes de ce groupe de Nématodes. Dans le tableau ci-dessus figurent, sur une première ligne, les hôtes prédominants pour chaque genre, Poissons d'eau douce ou Poissons marins; sur une deuxième ligne, se trouvent les migrateurs, qui sont très souvent des Salmonidés. Les Ascarophis, qui sont les parasites les plus fréquents chez les Poissons marins, ne paraissent pas exister chez d'autres migrateurs que l'Esturgeon. On connaît encore bien peu de cycles évolutifs de ces Spirurides permettant de relier leur présence à la biologie de leurs hôtes. On sait toutefois que Cystidicola farionis Fischer de Waldheim 1798 a pour hôte intermédiaire Gammarus pulex (L.) (Baylis 1931), Cystidicoloides harwoodi (A.-C. Chandler 1931), des nymphes d'Ephémères : Hexagenia recurvata Morgan et Polymitarcys sp. (Choquette 1955).

En ce qui concerne Ascarophis morrhuæ P. J. Van Beneden 1870 et Ascarophis filiformis Poljansky 1952, leur cycle a été découvert par A. V. Ouspenskaïa (1953, p. 828-831, fig. 1 : larve de filiformis, fig. 2 : larve de morrhuæ, fig. 3 : cycle de filiformis, fig. $4:$ cycle de morrhuæ), dans le Sud de la Mer de Barents. Les hôtes sont des Crustacés Décapodes; pour filiformis, ce sont: Hetairus polaris (Sabine 1824), Eualus gaimardi (H. M. Edwards 1837) et rarement Spirontocaris spinus (James Sowerby 1806); pour morrhuæ, ce sont : Eupagurus pubescens (H. Kröyer 1838) (jusqu'à 11 larves par hôte) et, plus rarement, Spirontocaris spinus (James Sowerby 1806), Hetairus polaris (Sabine 1824) et Pandalus borealis $\mathrm{H}$. Kröyer 1838. Les Crustacés s'infestent en ingérant les œufs libérés dans la mer avec les déjections des Morues et des Aiglefins. Chez ces Crustacés, la larve présente déjà les caractères de l'adulte, sauf pour l'appareil génital : les glandes génitales, les spicules, l'utérus ne sont pas encore développés et les sexes ne sont pas distincts. A. V. Ouspenskaïa a remarqué que ces Crustacés ne sont parasités par des larves d'Ascarophis que dans les eaux libres de la mer ouverte et dans les détroits menant aux baies; mais ne le sont pas dans le fond des baies près des rivages, parce que les hôtes définitifs adultes : Gadus morhua (L.) et Melanogrammus æglefinus (L.), n'y venant pas, l'infestation ne peut avoir lieu. Les alevins qui fréquentent le fond des baies près des rivages n'ont pas la même alimentation que les adultes et ne sont pas parasités par des Ascarophis.

On voit que ces hôtes sont très différents les uns des autres; il serait intéressant de poursuivre l'étude des cycles pour savoir si, dans un même genre, les hôtes intermédiaires sont voisins ou non. 
Du point de vue évolutif, on peut supposer que le type primitif était parasite de Poissons d'eau douce. L'apparition de Poissons migrateurs dans le cycle a permis la dissémination en eau saumâtre de certaines lignées qui ont pu, soit conserver des affinités étroites pour ce groupe d'hôtes particuliers, soit s'adapter à des hôtes exclusivement marins.

\section{RÉSUMÉ}

Il est décrit un nouvel Ascarophis, A. crassicollis, chez Gadus luscus L., différant principalement des autres espèces par un épaississement de la cuticule cervicale.

Une révision du genre est donnée, ainsi qu'une nouvelle définition et une clé de détermination pour les espèces suffisamment connues.

Les rapports avec les genres voisins sont étudiés, le genre Cystidicoloides M. S. Skinker 1931 est rétabli, une liste des espèces rattachées à Cystidicoloides et Metabronema est donnée.

La validité de ces genres est discutée et les meilleurs caractères différentiels sont étudiés.

\section{BIBLIOGRAPHIE}

Baylis (H. A.), 1931. - Gammarus pulex as an Intermediate Host for Troutparasites. Ann. Mag. Nat. Hist., Sér. 10, VII, p. 431-435, fig. 1-4.

- 1933. - The Nematode Genus Ascarophis Van Beneden. Ann. Mag. Nat. Hist., Sér. 10, XI, p. 112-117, fig. 1.

- 1934. - Three Helminthological Notes. Ann. Mag. Nat. Hist., Sér. 10, XIV, p. 115-121.

- 1935. - Four new species of Nematodes. Ann. Mag. Nat. Hist., Sér. 10, XVI, p. 370-382, fig. 1-8.

Campana-Rouget (Y.), 1955, - Sur deux nouveaux genres de Spirurides parasites de Poissons ; discussion systématique des genres voisins. Ann. Parasit. Hum. et Comp., XXX, $\mathrm{n}^{\circ} 4$, p. 346-362, fig. 1A-5B.

Chandler (A. C.), 1931. - New genera and species of Nematode Worms. Proc. U.S. Nat. Mus., LXXVIII, Art. 23, p. 1-11, pl. I, fig. 1-3, pl. II, fig. 4-10.

Chrтwoon (B. G.), 1934. - Two new Nematodes. Smithsonian Miscell. Coll., XCI, $\mathrm{n}^{\circ} 11$ (publication 3.243), p. 1-4, pl. fig. 1-10.

- et Chrtwood (M. B.), 1950. - An Introduction to Nematology. Sect. I, 213 p., fig. $1-145$ NN. Baltimore, $4^{\circ}$.

- et WeHR (E. E.), 1934. - The value of cephalic structures as characters in Nematode classification, with special reference to the superfamily Spiruroidea. Zeitsch. f. Parasit., VII, n 3, p. 273-335, fig. 1-20, pl. I. 
Choquette (L. P. E.), 1948. - On the species of the Genus Metabronema Y. and M. 1926, parasitic in Trout and Char. Canad. J. Res., D. XXVI, p. 329333, fig. 1-2.

- 1951. - Description of Metabronema prevosti sp. nov. With a note on the genus and a list of its species and their host and geographical distribution. Canad. Journ. Zool., XXIX, p. 102-108, fig. 1-2.

- 1955. - The Life-history of the Nematode Metabronema salvelini (Fujita, 1920) parasitic in the speckled Trout, Salvelinus fontinalis (Mitchill), in Quebec. Canadian Journ. of Zoology, t. XXXIII, p. 1-4, fig. 1-5.

Совв (N. A.), 1928. - The screw-nemas, Ascarophis Van Beneden 1871 ; parasites of codfishs, haddock and other fishes. J. Wash. Acad. Sci., XVIII, 4 , p. $96-102$, fig. $1-8$.

Dogifl (V. A.), 1932. - Eine neue in Acipenseriden parasitierende Nematodengattung aus der Familie Acuariidr. Zool. Anz., XCIX, 9-10, p. 263-269, fig. 1-4.

Foukment (L.), 1883. - Sur les filaments ovulaires chez les Nématodes. C.R. Soc. Biol., XXXV, p. 575-578.

1884. - Note sur un Nématode nouveau parasite du Merlan. Ann. Sci. Nat. Zool. (6 ${ }^{\circ}$ s.), XVII, art. 5, p. 1-8, pl. XVI, fig. 1-11.

Fusita (Tsunenobu), 1922. - On the Parasites of Japanese fishes. IV. Dobutsu Gaku Zasshi, XXXIV, n ${ }^{\circ} 403$, p. 292-300, fig. 1-8.

1928. - Further studies on Nematodes from Fishes of Lake Biwa. Dobutsu Gaku Zasshi, XL, n 477 , p. 303-314, fig. 1-3.

1939. - On the Nematoda parasites of the Pacific Salmon. Jour. Fac. Agric. Holkaido, XLII, n ${ }^{\circ}$, p. 239-266, pl. X-XIV, fig. 1-43.

1940. - Further notes on Nematodes of Salmonoid Fishes in Japan. Jap. J. Zool., VIII, n ${ }^{\circ} 4$, p. 377-394, fig. 1A-12B.

Gondon (A. R.), 1951. - On the male of Ascarophis morrhue Van Ben. Parasitol., XLI, 3-4, p. 261-263, fig. 1-3.

Johnston (T. H.) et Mawson (P. M.), 1944. - Remarks on some parasitic Nematodes from Australia and New Zealand. Trans. Roy. Soc. S. Austr., LXVIII, p. 60-66, fig. 1-13.

- 1945. - Some parasitic Nematodes from South Australian marine Fish. Trans. Roy. Soc. S. Austr., LXIX, n 1, p. 114-117, fig. 1-8.

1945. - Parasitic Nematodes. B.A.N.Z.A.R. Exped., 1929-31, ...Reports, series B, vol. V, Part 2, p. 75-159, fig. 1-51.

1947. - Some avian and fish Nematodes, chiefly from Tailem Bend, South Australia. Rec. S. Austr. Mus., VIII, n 4, p. 547-553, fig. 1-7.

Marкowsкi (St.), 1938. - Ueber die Helminthenfauna der baltischen Aalmutter (Zoarces viviparus L.). Zoolog. Polonia, III, p. 89-104, fig. texte 1A-5B, pl. I, fig. 1-3, pl. II, fig. 4-6.

Nicole (W.), 1907. - A Contribution towards a knowledge of the Entozoa of British marine Fishes, Part I. Ann. Mag. Nat. Hist., Sér. 7, XIX, p. 6694, pl, I-IV, fig. 1-16.

Olsen (Leland S.), 1952. - Some Nematodes parasitic in Marine fishes. Public. Inst. Marine Sci., Univ. of Texas, II, $\mathrm{n}^{\circ}$ 2, p. 172-215, pl. I-IV, fig. 1-84.

Ouspenskaĩ (A. V.), 1953. - Cycle évolutif du Nématode du genre Ascarophis Van Beneden (Nématodes Spirurata). Zoologitchesky Journal, Moscou, t. XXX, fasc. 5, p. 828-832, fig. 1-4. 
PolJansky (G. I.), 1952. - Quelques Nématodes nouveaux et peu connus parasites de l'intestin de Poissons marins. (En russe). Troudi Zoologitch. Inst. Moscou, XII, p. 133-147, fig. 1 (1)-4 (5).

Skinker (M. S.), 1931. - Three new parasitic nematode worms. Proc. U.S. Nat. Mus., LXXIX, art. 24, p. 1-9, fig. 1-19.

- 1931. - A redescription of Cystidicola stigmatura (Leidy), a Nematode parasitic in the swim-bladder of Salmonoid Fishes, and a new Nematode genus. Trans. Amer. Microsc. Soc., L, p. 372-375, pl. XLI-XLII, fig. 1-8.

SMedley (E. M.), 1934. - Some parasitic Nematodes from Canadian Fishes. J. Helm., XII, p. 205-220, fig. 1-14.

TAYLor (E. L.), 1925. - Notes on some new Nematodes in the Museum of the Liverpool School of Tropical Medicine. II. Ann. Trop. Med. and Parasit., XIX, p. 57-69, fig. 1-12.

Travassos (L.), Artigas (P.) et Pereira (Cl.), 1928. - Fauna Helminthologica dos peixes de agua doce do Brasil. Arch. Inst. Biol., I, p. 5-68, pl. I-XIV, fig. 1-155.

Tubangui (M. A.) et Masiluñgan (V. A.), 1938. - Nematodes in the Collection of the Philippine Bureau of Science. III. Philippine J. Sci., LXIV, n ${ }^{\circ} 3$, nov. 1937, p. 257-267, pl. I-III [a paru en 1938].

Uspenskaïa (A. V.). - Voir Ouspenskaĩa.

VAN Beneden (P. J.), 1870. - Les Poissons des côtes de Belgique, leurs parasites et leurs commensaux. Mém. Acad. Roy. Sci. Belg., XXXVIII, xx p. + 100 p., pl. I-VIII.

Yamaguti (S.), 1935. - Studies on the Helminth Fauna of Japan. Part 9. Nematodes of Fishes, I. Jap. J. Zool., VI, n 2, p. 337-386, fig. 1-65. 